Original Article

DOI: 10.15586/jomh.v16i3.255

\title{
A QUESTIONNAIRE SURVEY ON AWARENESS OF REPRODUCTIVE PROTECTION AND AUTOLOGOUS SPERM PRESERVATION AMONG CANCER PATIENTS
}

Wang $\mathrm{Xi}^{1}$, Dong Gan², Shao Cuihua ${ }^{3}$, Wang Peitao ${ }^{4}$

${ }^{1}$ International Hospital Preparatory Office, The Affiliated Hospital of Qingdao University, Shandong, China

${ }^{2}$ Shandong Provincial Third Hospital, Cheeloo College of Medicine, Shandong University, Shandong, China

${ }^{3}$ Department of Obstetrics, The Affiliated Hospital of Qingdao University, Shandong, China

${ }^{4}$ Department of Andrology, The Affiliated Hospital of Qingdao University, Shandong, China

Corresponding author: Wang Peitao: peitaowang@yeah.net

Submitted: 13 April 2020. Accepted: 29 June 2020. Published: 17 July 2020.

ABSTRACT

\section{Objective}

The aim of this study was to assess the awareness of fertility protection among male patients and their healthcare providers in the treatment process of cancer.

\section{Methods}

In a cross-sectional study, we applied a questionnaire survey among patients and healthcare providers who were selected randomly: the physician group consisted of oncologists and hematologists, and the patient group included participants with cancer from 15 to 45 years old, who were recruited from hospitals in Qingdao. Meanwhile, data from male cancer patients, who received sperm cryopreservation in our hospital, were also analyzed.

\section{Results}

Data collected from healthcare provider group showed that more than $80 \%$ of clinicians warned patients of the fertility impairment along with chemotherapy or other treatments, whereas a few clinicians recommended specific methods for reproductive protection to patients with special attention be paid to the follow-up fertility result. In patient group, more than $50 \%$ indicated they would accept the loss of fertility caused by cancer therapies. The rate of awareness of sperm cryopreservation, as an effective way of reproductive protection, was low among both physician and patient groups. In the past 3 years, only 63 samples of semen from 54 cancer patients were cryopreserved in our hospital. 


\section{Conclusion}

The awareness of reproductive protection in both physicians and cancer patients was not satisfied in Qingdao, China, and this finding indicates a need for comprehensive health education and practical protocols for both patients and healthcare providers in terms of reproductive protection for male patients with cancers.

Key Words: cancer; fertility impairment; reproductive protection; sperm cryopreservation

\section{INTRODUCTION}

Survival rate from cancer treatments has been increased. Even over $80 \%$ of young cancer survivors could live a high-quality life after successful procedures, rather than just extending life span., ${ }^{1,2}$ When being diagnosed with cancer, young childless patients tend to have stronger desire for a normal physical condition, enabling them to rise their own children after treatment. However, harm to fertility from cancer therapies can be permanent, and corresponding treatments to cancer, as well as to other severe chronic diseases, usually cause infertility or premature gonadal insufficiency. ${ }^{3}$ In recent years, knowledge on various fertility preservation (FP) options to be discussed with young cancer patients whose fertility might be at risk has been increased before gonadotoxic therapy. However, in China, there is not adequate data on fertility impairment and reproductive protection regarding cancer treatment. Sperm cryopreservation, as an essential technique of human sperm bank, is also utilized in assisted reproductive technology (ART) for male FP in clinical practice. With the development and wide application of ART, sperm cryopreservation has gradually become the main component of ART. ${ }^{4}$

In this report, we acquired data to assess the current status of fertility impairment and reproductive protection among male cancer patients by two questionnaire surveys delivered separately in physician group and patient group in Qingdao, China. Meanwhile, we also analyzed the data of patients who received sperm cryopreservation in our hospital in the past 3 years.

\section{MATERIALS AND METHODS}

\section{Subjects and Questionnaire of Cross-Sectional Study}

\section{Patient group}

Patients who met the following criteria were enrolled to the study: inpatient or outpatient male cancer patients, aged from 15 to 45 years old, and voluntary participation in the study. The exclusion criteria were as follows: patients with psychological diseases or other significant diseases, childbearing difficulties, medical disputes, and so on.

\section{Physician group}

Oncologists and hematologists from five local hospitals from Qingdao city were selected randomly to participate in this study.

\section{Questionnaire}

Specific questionnaires were stated in the result part. All the participants fully understand the purpose of this survey to ensure the validity of the data; to achieve this, responders were allowed to question freely based on material we provided before signing a consent form. The entire study process had been approved by the ethic committee of the Affiliated Hospital of Qingdao University.

\section{Analysis of Semen Cryopreservation in Our Hospital}

Sample collection and analysis tool

From 2015 to 2018, our department had conducted 63 cases of autologous semen cryopreservation for 54 patients. The semen samples 
were collected before cancer treatment by masturbation after abstinence of 3-5 days, and assessed based on the Sperm Analysis Manual of WHO. The parameters of sperm were analyzed by Computer-Assisted Sperm Analysis (CASA), mainly including sperm production amount, sperm concentration, and motility rate.

Semen cryopreservation and thawing process

A prestep of semen cryopreservation is semen samples assessment. We applied the sperm-freezing medium (Quinn's advantage) as a guide for freezing protocol. The general steps include the following: first, suspend semen with sperm-medium at a ratio of $1: 1(\mathrm{~V} / \mathrm{V})$, separate the mixture into several aliquots with each amount of $0.8-1.0 \mathrm{~mL}$, and then put into cryovials separately; second, place cryovials in refrigerator with constant temperature of $4^{\circ} \mathrm{C}$ for $20 \mathrm{~min}$; thirdly, move the cryovials into freezer with $-80^{\circ} \mathrm{C}$, store overnight, and then soak the cryovials in liquid nitrogen for long-term preservation. Throughout the whole process, about $0.5 \mathrm{~mL}$ of the mixture was cryopreserved and thawed on the second day to evaluate the sperm quality by CASA.

Semen thawing: take the cryopreserved semen sample out from liquid nitrogen and let stand at room temperature (about $22^{\circ} \mathrm{C}$ ) until a small ice ball remained. Then the semen sample is ready to be used in ART.

\section{Data from patients}

We use these 54 patients as a sample to speculate the source of knowledge and concerns regarding semen cryopreservation in general male cancer patients by two questions, which will be described later in this article.

\section{RESULTS}

\section{Patient Group}

Questionnaire results

A total of 500 copies of survey were distributed among outpatients and inpatients in oncology departments from five hospitals in Qingdao, whereas 466 have been collected back, from which 407 copies were valid and summarized in Table 1.

\section{Physician Group}

\section{Questionnaire results}

Eighty clinicial specialists, including mostly oncologists and hematologists, completed the survey, from which 76 copies were validated. The specific questions and results are presented in Table 2.

\section{The physicians' focuses}

Where do you pay the most attention when considering treatment protocols? There do exist different opinions among physicians. The result of this questionnaire is as follows - curative effect: $32.9 \%$, physical endurance of patients: $27.6 \%$, side effects: $21.1 \%$, life quality of patients after

TABLE 1 Contents and Results of Questionnaire for Patients

\section{Questions/Answers}

1. Did your doctors inform you of fertility impairment and reproductive protection? (Yes/No)=328/79 (80.6\%)

2. Have you ever considered your future fertility and felt anxious about it? (Yes/No)=333/74 (81.8\%)

3. Would you accept the fertility damages or loss due to cancer treatment? (Yes/No)=254/153 (62.4\%)

4. Did your doctor recommend autologous semen cryopreservation to you before cancer treatment when necessary? $($ Yes/No) $=255 / 152(62.7 \%)$

5. Would you accept any new methods of reproductive protection from your doctors? (Yes/No)=352/55 (86.5\%) 
TABLE 2 Contents and Results of Questionnaire for Physicians

Questions/Answers
1. Have you ever considered the future fertility of your patients in the past? (Yes/No $)=(69 / 7)=91.4 \%$
2. Do you think it is necessary for patients to protect or preserve fertility before treatment? $($ Yes $/ \mathrm{No})=(74 / 3)=97.1 \%$
3. Have you informed your patients about fertility impairments caused by treatment? $($ Yes $/ \mathrm{No})=(69 / 7)=91.4 \%$
4. Have you assessed your patients' fertility before and after treatment? (Yes/No)=(39/38)=51.3\%
5. Have you given enough attention to fertility impairment and attempted to avoid it when making treatment
protocol? (Yes/No) $=(69 / 7)=91.4 \%$
6. Have you recommended autologous semen cryopreservation for those at childbearing age?
(Yes/No) $=(46 / 30)=56.6 \%$
7. Have you informed your patients about fertility following-up at the end of treatment? $($ Yes $/ \mathrm{No})=(24 / 52)=31.6 \%$

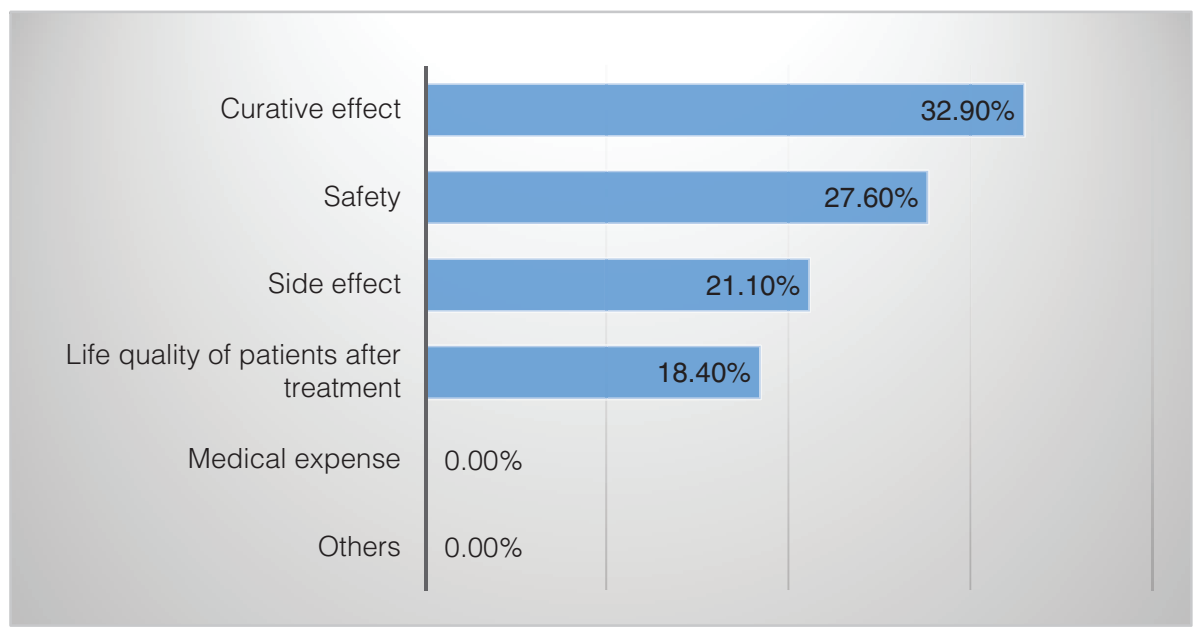

FIG. 1 Physicians' focus.

treatment: $18.4 \%$, medical expense: $0.0 \%$, and others: $0.0 \%$. The results are also shown in Figure 1.

Physicians' preference on reproductive protection methods

There are many reproductive protection methods with various effects. Oncologists are supposed to provide suitable recommendations to patients before the treatment. With regard to the question asking about physicians' selection preference on reproductive protection methods toward patients with cancer, the contents and results are as followed and showed in Figure 4. The results are also shown in this figure, $61.8 \%$ chose semen cryopreservation before treatment, $19.7 \%$ chose avoiding radiation, $10.5 \%$ chose safety and less toxic chemotherapy, and 7.9\% chose comprehensive treatment (see Figure 2).

\section{Semen Cryopreservation in Our Hospital}

\section{Patients' characteristics}

We had been collecting data for the past 3 years from 54 patients aged from 15 to 40 years old, who accepted autologous semen cryopreservation in our department before any cancer therapies. Details on patient's characteristics are listed as follows. 


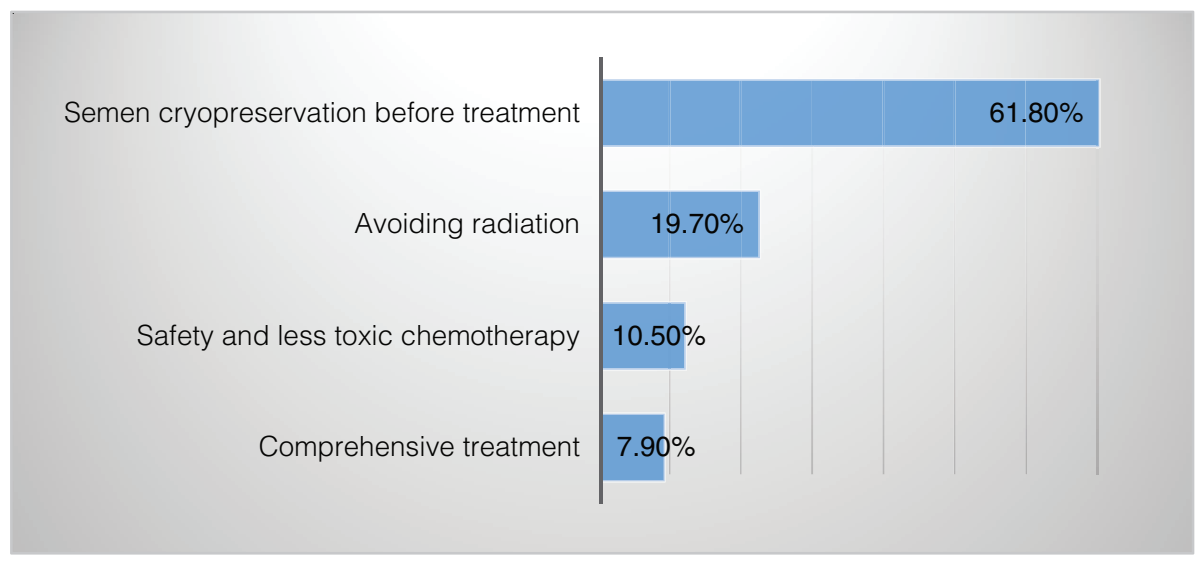

FIG. 2 Physicians' preference on reproductive protection.

- Age distribution

- <20 years old: 6 individuals

- 20-30 years old: 32 individuals

- 30-40 years old: 16 individuals

- Marital and reproductive status

- Single: 18 individuals

- Married and childless: 6 individuals

- Married and having children: 30 individuals

- Disease diagnosed

- Lymph cancer: 14 individuals

- Testiculoma: 8 individuals

- Leukemia: 26 individuals

- Others: 6 individuals

- Treatment protocols

- Chemotherapy: 28 cases

- Chemotherapy and radiotherapy: 12 cases

- Surgery and radiotherapy/ chemotherapy: 14 cases

- Education

- Secondary education and lower: 6 individuals $(11.1 \%)$

- University and above: 48 individuals $(88.9 \%)$
Semen cryopreservation and usage

Those 54 patients had provided 63 cases of autologous semen cryopreservation in total including repetitive operation. Up to now, preservation period of these cases is from 8 months (due to patient's death) up to 68 months. Ten samples from eight patients, with an average cryopreservation period of 28 months, were used for ART. The usage rate is $16 \%$ (10\63). Three samples from two patients were disposed due to patients' death, of which the average period was 13 months.

\section{Questionnaire to the patients}

A simple questionnaire survey was conducted among 54 patients to assess their source of knowledge about semen preservation and concerns when considering semen cryopreservation. Doctor's recommendation takes the main role when it comes to patients' source of knowledge, and followed by active consultation and social media [see Figure 3. Source of knowledge on semen preservation (single choice)]. When considering an actual practice of semen preservation, there are several aspects that patients care more, such as economic factors, feasibility, safety, and so on [see Figure 4. Concerns on semen cryopreservation (multiple choice)]. 


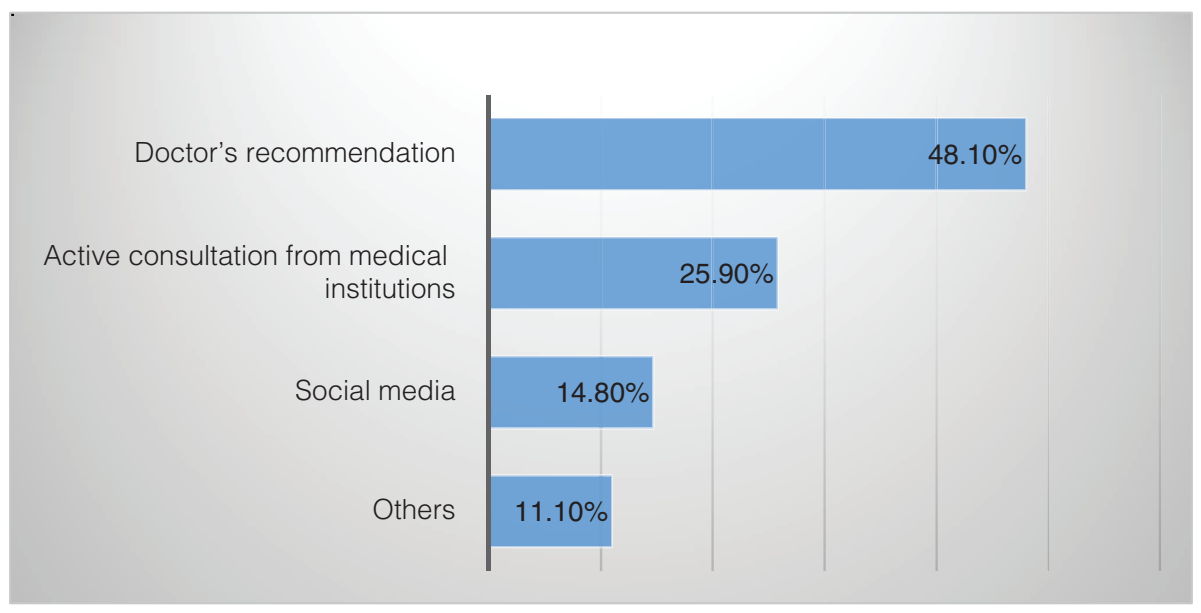

FIG. 3 Source of knowledge on semen preservation (single choice).

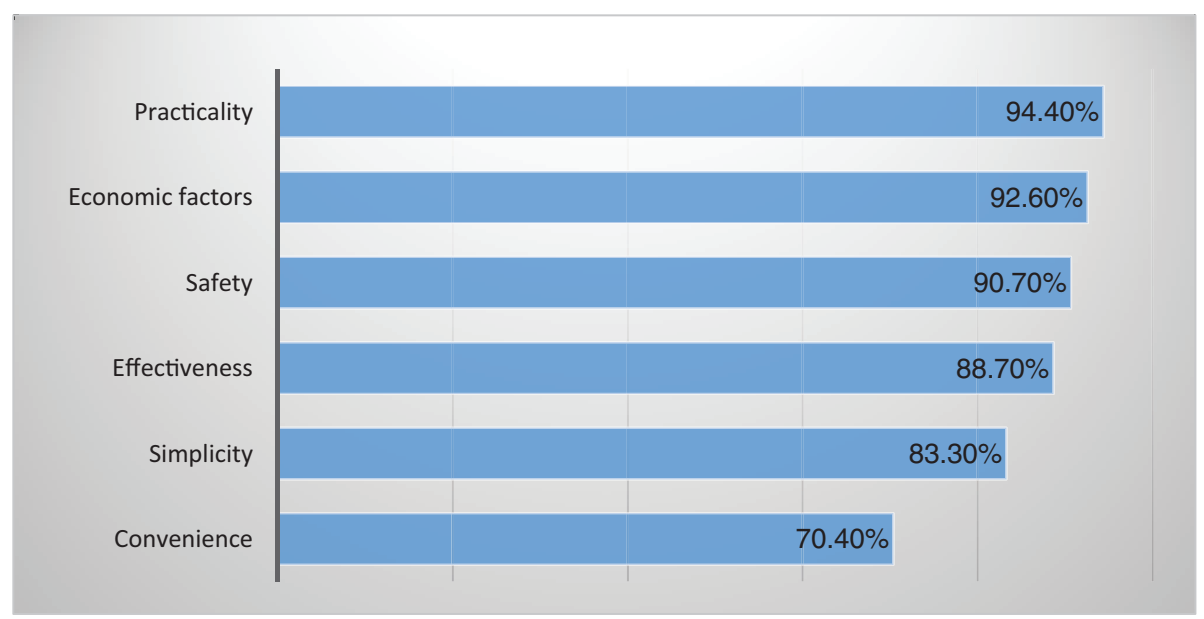

FIG. 4 Concerns on semen cryopreservation (multiple choice).

\section{DISCUSSIONS}

\section{Current Applying Situation and Need of Reproductive Protection}

Any change or abnormity of microenvironment in testis will interfere the normal proliferation and differentiation of gonad cells, especially the germ cells, negatively influencing sperm production and subsequent fertility. As the most commonly used and effective cancer treatment procedure before and after operation, chemotherapy would disable, damage, or impair the functioning of gonads, causing infertility or subfertility among patients of childbearing age, which may also cause serious mental distress to the patients. ${ }^{5,6}$

Survival rate of cancer is increasing rapidly along with the improvement of medical technologies; however, patients at childbearing age concern more and more on fertility along with life 
expectancy. Unfortunately, fertility impairment or damage might be lasting, that is defined as subfertility or infertility. Studies on survivors of cancers reported a long-term psychological distress associated with the infertility after treatment, led by a missed opportunity for FP before treatment due to the absent discussion of related consequences. Thus, sufficient communication on reproduction protection before and during cancer treatment is significantly important, and the most effective and direct action to protect fertility and preserve reproductive opportunities for cancer patients, allowing them raising their own children. ${ }^{7,8}$

Indeed, negligence to this issue happens to both physicians and patients when more attention is focused on the best curative effect in choosing treatment protocols. This article is assessing the awareness on reproductive protection among both patients and physicians. The results indicated that most physicians could warn patients about the risk of fertility impairment due to cancer therapies, and were able to design treatment plan prudently. Nonetheless, only $50-60 \%$ of doctors delivered fertility assessment and reproduction protection plans to patients.

\section{Reproductive Protection Techniques}

Nowadays, reproductive protection has a consideration of cancer treatment in three major aspects to protect or preserve patients' fertility. They are selection of hypotoxic chemotherapy protocols to gonad, reducing sensitivity of gonad by endocrine therapy to gonad axis before treatment, and diminishing side effects by medication. Proliferative and differential germ cells are extremely vulnerable to oxidative stress, and antioxidants have been proven to resist the oxidative stress damage caused by chemical drugs. ${ }^{1-4}$

Although there are various reproductive protection strategies, sperm cryopreservation, as a well-established cell cryopreservation technique, is the most practical and effective FP method. It is also a main component of artificial reproductive technology (ART) and widely used around the world. 5,9

\section{Feasibility Semen Cryopreservation}

According to our result, semen cryopreservation is a simple and practicable method for FP, but not be widely used in clinical settings yet. However, it is a common technology in field of ART. ${ }^{10-12}$ Bunge and Sherman reported the first successful case of artificial insemination with cryopreserved semen in $1953 .{ }^{3}$ Studies after that have confirmed that motility and fertility of sperm can be maintained for several decades when cryopreserved and stored in liquid nitrogen. Theoretically, sperm can be stored in liquid nitrogen $\left(-196^{\circ} \mathrm{C}\right)$ permanently. With the innovation of technology, reproductive medicine specialists and cryobiologists invented cryopreservation techniques for one single or several sperm cells for intracytoplasmic sperm injection (ICSI).

\section{Current Challenges and Coping Strategies}

From the data, the unsatisfactory awareness on FP among cancer patients is mainly caused by neglecting such issue by physicians when they only focus on therapeutic effect instead of an overall consideration. Most patients in our study did semen cryopreservation before treatment following their doctor's advice, which confirmed a good compliance from patients to doctors. Thus, to raise doctors' awareness on patient education of reproductive protection is a significant prerequisite to increase patients' awareness.

In a study by Rabah, $64.1 \%$ physicians attached importance of sperm cryopreservation, from which $88 \%$ thought that cryopreserving sperm before treatment is necessary for patients, whereas only $41.7 \%$ actually delivered their recommendation to patients as routine. ${ }^{13}$ From the patients' side, a questionnaire among 283 male patients reported that $80 \%$ were informed about fertility damage by treatment from doctors. ${ }^{14}$ Most physicians in our study did concern on fertility damage of patients and were able to take reproductive 
protection into account before and during treatment. They were reported to be willing to discuss potential harm caused by therapy with patients, but failed to provide practical and concrete reproduction protection or FP options.

In France, a freezing and storing network composed of 23 sperm banks has been established..$^{15}$ In the United States, the Community Tumor Research Plan of National Cancer Institute established a systematic reproductive protection for patients. ${ }^{16}$ In China, until 2017, 26 qualified sperm banks had been authorized to conduct sperm cryopreservation for patients or donors. However, autologous sperm cryopreservation just made up a small proportion in the entire sperm cryopreservation in China.

Reported by a sperm bank in Zhejiang Province, in a 8-year period from 2005 to 2013, there were only 43 cases of autologous sperm cryopreservation from cancer patients. ${ }^{17} \mathrm{~A}$ reproductive center of a hospital in Hong Kong reported only 110 autologous semen preservation cases for cancer patients in the past 17 years. ${ }^{18}$ An investigation did in Shanghai reported only $21.6 \%$ male cancer patients actually did autologous semen cryopreservation before treatment under doctors' advice. ${ }^{19}$ All above studies reveal a fact that autologous semen cryopreservation has great potential in clinical application, which coincides with our findings that $86.5 \%$ of our patients would like to accept new fertility protection methods when being asked. . $^{13,14}$

As we mentioned previously, patients' low awareness on semen cryopreservation is mainly due to the deficient of patient education from healthcare providers. And the ultimate psychological pressure to the patients is on the long run, from which we can rule out the possibility of using donated sperm to produce offspring by cancer patients.

Currently, relevant professional organizations, such as American Society of Clinical Oncology (ASCO), European Society of Human
Reproduction and Embryology (ESHRE), and the Clinical Oncology Society of Australia (COSA), all recognized the importance of FP to cancer patients, and mandated requirements on such patient education conversation in their guidelines. ${ }^{15,16}$

\section{CONCLUSION}

In this study, we analyzed results of questionnaires to assess the awareness on fertility impairments and reproductive protection caused by cancer treatment among both physician and patient groups. Meanwhile, we also discussed implementation of autologous sperm cryopreservation in our hospital and some sperm banks. It indicates that autologous sperm cryopreservation is rarely practiced due to low awareness on reproductive protection among both physicians and patients; even it is simple and effective.

There are several coping strategies we strongly recommend. First, treatment protocol with risk of fertility impairments should include a holistic FP plan delivered by a MDT (multidisciplinary team) consisting of doctors, scientists, nurses, counselors, administrators, and researchers. Such professional team should have capacity to cope with acute consultation and provide a long-term follow-up service. Second, all members from the MDT need to be trained adequately on reproductive protection techniques and related knowledge. Third, sufficient patient education should be implemented as routine by chief physicians or nurses, including enough information allowing patients to fully understand possible risk of fertility damages and reproductive protection options to empower them on treatment decision-making. ${ }^{9,20}$

\section{CONFLICT OF INTEREST}

The authors declare no conflicts of interest. 


\section{FUNDING}

No funding was received for this work.

\section{REFERENCES}

1. Tournaye H, Dohle GR, Barratt CLR. Fertility preservation in men with cancer. Lancet 2014;84:1295-301. https://doi.org/10.1016/S01406736(14)60495-5

2. Karunakaran P, Malhotra P, Lad D. Fertility management for the hemato-oncologist. Indian J Hematol Blood Transfus 2018; 34(1):13-18. https://doi.org/10.1007/s12288-01 7-0887-8

3. Bunge RG, Sherman JK. Fertilizing capacity of frozen human spermatozoa. Nature 1953; 172(4382):767-8. https://doi.org/10.1038/17276 $7 \mathrm{~b} 0$

4. Kawai K, Nishiyama H. Preservation of fertility of adult male cancer patients treated with chemotherapy. Int J Clin Oncol. 2019;24:34 40. https:// doi.org/10.1007/s10147-018-1333-0

5. Yogev L, Kleiman SE, Shabtai E, et al. Long-term cryostorage of spermin a human sperm bank does not damage progressive motility concentration. Hum Reprod 2010;25(5):1097-103. https://doi. org/10.1093/humrep/deq041

6. Okada K, Fujisawa M. Recovery of spermatogenesis following cancer treatment with cytotoxic chemotherapy and radiotherapy. World J Mens Health. 2019;37(2):166-174 https://doi.org/ 10.5534/wjmh. 180043

7. Mancini J, Rey D, Préau M, et al. Infertility induced by cancer treatment: Inappropriate or no information provided to majority of French survivors of cancer. Fertil Steril 2008;90(5):1616 25. https://doi.org/10.1016/j.fertnstert.2007.08. 064

8. Moody JA, Ahmed K, Yap T, Minhas S, Shabbir M. Fertility management in testicular cancer: The need to establish a standardized and evidence-based patient-centric pathway. BJU Int 2019;123:160-72. https://doi.org/10.1111/bju.14455

9. Stern C, Agresta F. Setting up a fertility preservation programme. Best Pract Res Clin Obstet
Gynaecol 2019;55:67-78. https://doi.org/10.1016/ j.bpobgyn.2018.07.007

10. Horne G, Atkinson AD, Pease EH, et al. Live birth with spermcryopreserved for 21 years prior to cancer treatment: Case report. Hum Reprod 2004;19(6):1448-9. https://doi.org/10.1093/humrep/deh249

11. Feldschuh J, Brassel J, Durso N, et al. Successful sperm storage for 28 years. Fertil Steril 2005;84(4):1017. https://doi.org/10.1016/j.fertnstert.2005.05.015

12. Liu F, Zou SS, Zhu Y, et al. A novel micro-straw for cryopreservationof small number of human spermatozoon. Asian J Androl 2016. https://doi. org/10.4103/1008-682X.173452

13. Rabah DM, Wahdan IH, Merdawy A, et al. Oncologists' knowledge andpractice towards sperm cryopreservation in Arabic communities. J Cancer Survive 2010;4(3):279-83. https://doi. org/10.1007/s11764-010-0140-z

14. Shnorhavorian M, Harlan LC, Smith AW, et al. Fertility preservationknowledge, counseling, and actions among adolescent and young adultpatients with cancer: A population-based study. Cancer 2015;121(19):3499-506. https://doi. org/10.1002/cncr.29328

15. Daudin M, Rives N, Walschaerts M, et al. Sperm cryopreservation inadolescents and young adults with cancer: Results of the French national sperm banking network (CECOS). Fertil Steril 2015,103(2):478-86. https://doi.org/10.1016/j. fertnstert.2014.11.012

16. Besharati M, Woodruff T, Victorson D. Young adults' access to fertility preservation services at National Cancer Institute Community Oncology Research Program minority/underserved community sites: Aqualitative study. J Adolesc Young Adult Oncol 2016;5(2):187-200. https://doi. org/10.1089/jayao.2015.0034

17. Sheng HQ, Zhang XZ, Hong Y. Analysis of the quality of cryopreserved semen from male cancer patients. Natl J Androl 2015;21(1):44-7.

18. Chung JP, Haines CJ, Kong GW. Sperm cryopreservation for Chinese male cancer patients: A 17-year retrospective analysis in an assisted reproductive 
unit in Hong Kong. Hong Kong Med J 2013;19(6): 525-30. https://doi.org/10.12809/hkmj134055

19. Ping $\mathrm{P}, \mathrm{Gu} \mathrm{BH}, \mathrm{Li} \mathrm{P}$, et al. Fertility outcome of patients with testiculartumor: Before and after treatment. Asian J Androl 2014;16(1):107-11. https://doi.org/10.4103/1008-682X.122194
20. Traila A, Dima D, Achimas-Cadariu P, Micu R. Fertility preservation in Hodgkin's lymphomapatients that undergo targeted moleculartherapies: An important step forward fromthe chemotherapy era. Cancer Manage Res 2018;10:1517-26. https:// doi.org/10.2147/CMAR.S154819 